Original Paper http://ajol.info/index.php/ijbcs http://indexmedicus.afro.who.int

\title{
Réponse du sésame (Sesamum indicum L.) à l'inoculation mycorhizienne avec des souches des champignons mycorhiziens arbusculaires indigènes du Burkina Faso
}

\author{
Hadou HARO* et Kadidia B. SANON \\ Laboratoire de Microbiologie, INERA/DEF BP 7047 Ouagadougou 03, Burkina Faso. \\ *Auteur correspondant ; E-mail : harohadou@yahoo.fr, Tel :(00226)76627083.
}

\section{RESUME}

Au Burkina Faso, le sésame est essentiellement une culture de rente. Sa production augmente progressivement alors que son rendement baisse d'année en année. Ceci est lié à la pauvreté du sol en éléments minéraux tels que l'azote et le phosphore assimilable entrainant une augmentation des superficies emblavées pour cette culture et une utilisation des intrants chimiques de plus en plus grande. Or les intrants chimiques ont montré leurs limites dans leur utilisation. C'est ainsi que cette étude a été initiée afin de contribuer à l'amélioration de la production agricole durable. Dans cette étude le sésame a été cultivé en serre et inoculé avec deux inocula mycorhiziens indigènes. Les paramètres de croissance ont été mesurés à 30 et 60 jours après semis. La biomasse aérienne, racinaire et totale ont été évaluées à 60 jours après semis. Les résultats montrent une amélioration de la croissance en hauteur de $431,25 \%$, taux de croissance relative en hauteur de 145,87\%, diamètre au collet de $163,92 \%$, biomasse aérienne de 102,9\%, biomasse racinaire de $126,67 \%$ et de la biomasse totale de $110,1 \%$ par l'inoculation. Cette étude a montré des résultats prometteurs et mérite d'être approfondie par des essais aux champs.

(C) 2020 International Formulae Group. All rights reserved.

Mots clés : Sésame, inoculation mycorhizienne, champignons mycorhiziens arbusculaires, Burkina Faso.

\section{Sesame (Sesamum indicum $\mathrm{L}$.) response to mycorrhizal inoculation with native arbuscular mycorrhizal fungi to Burkina Faso}

\begin{abstract}
In Burkina Faso, sesame is essentially a cash crop. Its production is gradually increasing while its yield decreases. This is linked to the soil poverty in mineral elements such as available nitrogen and phosphorus leading to an increase in the area sown for this crop and an increasing use of chemical inputs. However, chemical inputs have shown their limits in their use. This is how this study was initiated to help improve sustainable agricultural production. In this study, sesame was grown in greenhouse and inoculated with two inocula of native arbuscular mycorrhizal fungi (M1 and M2) from Burkina Faso. The growth parameters were measured at 30 and 60 days after sowing. Shoot, root and total biomass were assessed at 60 days after sowing. The results obtained show an improvement in height growth of $431.25 \%$, the relative growth rate in height of $145.87 \%$, the collar diameter of
\end{abstract}


$163.92 \%$, the aboveground biomass of $102.9 \%$, root biomass of $126.67 \%$ and total biomass of $110.1 \%$ of plants inoculated with arbuscular mycorrhizal fungi compared to the inoculated control. This study has shown promising results and deserves to be deepened by field tests to assess the effect of this inoculation on sesame yield.

(C) 2020 International Formulae Group. All rights reserved.

Keywords: Sesame, mycorrhizal inoculation, arbuscular mycorrhizal fungi, Burkina Faso.

\section{INTRODUCTION}

Le sésame (Sesamum indicum L.) est une plante oléagineuse alimentaire avec une valeur nutritionnelle importante (acides gras : 45 à $55 \%$, protéines : 19 à $25 \%$, vitamines : $\mathrm{B}$, E..., minéraux : $\mathrm{Ca}, \mathrm{K}, \mathrm{Mg}, \mathrm{P} . .$. ) (Boureima, 2007). Au Burkina Faso, il constitue essentiellement une culture de rente. C'est le deuxième produit agricole d'exportation du Burkina, derrière le coton et générant ainsi plus 350 milliards de FCFA par an. De ce fait, la production a connu une augmentation depuis 2010, passant de 90649 tonnes (2010) à 163787 tonnes (2017) (FAO, 2019). Cette augmentation est liée majoritairement à l'augmentation de la superficie emblavée qui passe de 125471 ha (2010) à 506095 ha (2014) puis à 291173 ha en 2017. Cependant, le rendement ne fait que décroitre depuis 2010 passant ainsi de $0,72 \mathrm{t} / \mathrm{ha}$ (2010) à $0,56 \mathrm{t} / \mathrm{ha}$ (2017) (FAO, 2019). Ceci serait lié à l'appauvrissement croissant des sols en éléments minéraux majoritairement l'azote et le phosphore, d'où la nécessité de développer des méthodes permettant d'améliorer durablement la fertilité des sols afin d'accroitre le rendement de cette culture. Or il est bien établi que le sésame forme une symbiose avec les champignons mycorhiziens arbusculaires (Diouf et al., 2009; Diatta et al., 2013; Askari et al., 2019) alors que l'effet de la symbiose mycorhizienne sur la croissance et la production des plantes n'est plus à démontrer (Haro et al., 2012a; Diatta et al., 2013; Haougui et al., 2013; Haro et al., 2015; Haro et al., 2016a; Haro et al., 2016b; Haro et al., 2017; Askari et al., 2019). L'exploitation de cette symbiose serait une possibilité pour améliorer la productivité du sésame. C'est ainsi que nous nous sommes intéressés à la symbiose mycorhizienne du sésame avec pour objectif d'évaluer sa réponse à l'inoculation mycorhizienne avec les champignons mycorhiziens arbusculaires indigènes du Burkina Faso.

\section{MATERIEL ET METHODES}

Matériel

Matériel végétal

Le matériel végétal était composé de graines de sésame couramment cultivées au Burkina Faso.

\section{Matériel fongique}

Le matériel fongique était composé de deux champignons mycorhiziens arbusculaires (CMA) locales efficients isolés de la rhizosphère du niébé cultivé au Burkina Faso (Haro, 2016) : complexe mycorhiziens [Scutellospora sp., Gigaspora sp., Glomus sp. (M1)] et Glomus sp. (M2).

\section{Substrat de culture}

Le substrat de culture était un sol stérilisé de Ouagadougou et ses caractéristiques physico-chimiques sont présentées dans le Tableau 1.

\section{Méthode \\ Traitement des graines}

Les graines de sésame ont été aseptisées par trempage dans l'éthanol $96^{\circ}$ pendant 3 minutes, rincées abondamment à l'eau distillée stérile puis dans une solution d'hypochlorite de calcium $\left(\mathrm{CaCl}_{2} \mathrm{O}_{2}\right.$ à $\left.3,3 \% ; \mathrm{p} / \mathrm{v}\right)$ pendant $3 \mathrm{~min}$ et rincées abondamment à l'eau distillée stérile. Ces graines ont été ensuite semées à raison de 4 par pot.

\section{Mise en place de l'essai}

L'essai a consisté à cultiver le sésame dans des pots de 2 litres contenant $2000 \mathrm{~g}$ de sol stérilisé de Ouagadougou (Tableau 1). Le dispositif expérimental utilisé est de type randomisé simple. Ce dispositif est composé de 
3 traitements [deux traitements inoculés (M1 et M2) et un témoin non inoculé)] et chaque traitement est répété 5 fois. Le sésame a été semé à raison de 4 graines par pot et le démariage est intervenu à la deuxième semaine après semis de sorte à laisser un plant par pot. Cette culture a duré 60 jours (stade de floraison/fructification).

Les inocula mycorhiziens ont été apportés au moment des semis. L'inoculum mycorhizien était constitué d'un mélange de spores, de propagules fongiques, de fragments de racines mycorhizées et de sol. L'inoculation mycorhizienne a consisté à l'apport de l'inoculum mycorhizien à raison de $10 \mathrm{~g}$ par pot pendant les semis.

Pour estimer la réponse du sésame à l'inoculation mycorhizienne, la hauteur, le diamètre au collet, le taux de croissance relative en hauteur et le taux de croissance relative du diamètre au collet ont été mesurés au $30^{\mathrm{e}}$ et au $60^{\mathrm{e}}$ jour après semis. La biomasse aérienne et racinaire ainsi que la fréquence et l'intensité de mycorhization ont été mesurées à la fin de l'essai (60 jour après semis).

Mesure de la hauteur, du diamètre au collet, du taux de croissance relative en hauteur et du taux de croissance relative du diamètre au collet

La hauteur a été mesurée à l'aide d'une règle graduée à 30 et 60 jours après semis. Le taux de croissance relative en hauteur (TCRh) est estimé selon la formule suivante :

$\mathrm{TCRh}=(\mathrm{Hf}-\mathrm{Hi}) / \mathrm{Hi}$ avec $\mathrm{H}:$ hauteur, $\mathrm{i}$ : initiale, $f$ : finale.

Le diamètre au collet est mesuré à l'aide d'un pied à coulisse au niveau de la zone de séparation entre le système racinaire et la partie aérienne à 30 et 60 jours après semis. Le taux de croissance relative du diamètre au collet (TCRdc) est estimé par la formule suivante :

TCRdc $=($ Dcf - Dci $) /$ Dci , avec Dc : Diamètre au collet, $\mathrm{i}:$ initiale et $\mathrm{f}: \mathrm{f}$ : finale.

Mesure de la biomasse aérienne, racinaire et totale

A 60 jours après semis, chaque plant a été dépoté soigneusement de façon à récupérer la partie aérienne et toutes les racines des plants. Toutes ces parties ont été séchées à l'étuve à $70^{\circ} \mathrm{C}$ pendant 72 heures pour la mesure de la biomasse aérienne, racinaire et totale. Après la mesure de la biomasse, les racines ont été utilisées pour l'étude de l'infection mycorhizienne.

\section{Mise en évidence de l'infection mycorhizienne}

La mise en évidence de l'infection endomycorhizienne a été réalisée par la coloration des racines fines des plants selon la méthode de Phillips et Hayman (1970) et l'observation au microscope photonique. Les racines fines des plants ont été découpées en fragments d'un centimètre et ont été colorées suivant la méthode décrite par Phillips et Hayman (1970). Les fragments colorés ont été montés entre lames et lamelles dans du glycérol. La fréquence et l'intensité de mycorhization ont été évaluées au microscope optique au grossissement 400 en utilisant la méthode de Trouvelot et al. (1986).

\section{Analyse statistique}

Toutes les données collectées pour les différents paramètres ont été soumises à une analyse de variance (ANOVA) avec le logiciel statistique XLSTAT 2018 et les moyennes des variables ont été comparées en utilisant le test de Newman Keuls au seuil de probabilité $\mathrm{p}=5$ $\%$.

Tableau 1 : Caractéristiques physico-chimiques du substrat de culture.

\begin{tabular}{|c|c|c|c|c|c|c|c|c|c|c|}
\hline $\begin{array}{c}\text { Substrat } \\
\text { de } \\
\text { culture }\end{array}$ & $\begin{array}{c}\text { Argile } \\
(\%)\end{array}$ & $\begin{array}{c}\text { Limons } \\
\text { totaux } \\
(\%)\end{array}$ & $\begin{array}{c}\text { Sables } \\
\text { totaux } \\
(\%)\end{array}$ & $\begin{array}{c}\text { Matière } \\
\text { organique } \\
\text { totale }(\%)\end{array}$ & $\begin{array}{c}\text { Carbone } \\
\text { total } \\
(\%)\end{array}$ & $\begin{array}{c}\text { Azote } \\
\text { total } \\
(\%)\end{array}$ & $\mathrm{C} / \mathrm{N}$ & $\begin{array}{c}\text { Phosphore } \\
\text { total en } \\
\text { ppm }\end{array}$ & $\begin{array}{c}\text { Phosphore } \\
\text { assimilable } \\
\text { en ppm }\end{array}$ & $\begin{array}{c}\text { pH } \\
\text { eau } \\
(\mathrm{p} / \mathrm{v}: \\
1 / 2,5)\end{array}$ \\
\hline & 3,92 & 5,88 & 90,2 & 0,331 & 0,192 & 0,016 & 12 & 172,52 & 1,74 & 6,44 \\
\hline
\end{tabular}




\section{RESULTATS}

Mesure de la hauteur, du taux de croissance relative en hauteur, du diamètre au collet et du taux de croissance relative du diamètre au collet du sésame

Le Tableau 2 présente les résultats des mesures de la hauteur, du taux de croissance relative en hauteur, du diamètre au collet et du taux de croissance relative du diamètre au collet du sésame inoculé avec des champignons mycorhiziens arbusculaires indigènes. $\mathrm{La}$ croissance du sésame varie en fonction de traitement. Les analyses statistiques montrent des différences significatives $(\mathrm{P}<0,05)$ entre les traitements inoculés et non inoculés pour tous les paramètres sauf pour le taux de croissance relative du diamètre au collet. Cependant, aucune différence significative $(\mathrm{P}>0,05)$ n'a été observée entre les traitements inoculés pour tous les paramètres. Globalement, la croissance du sésame a été améliorée par l'inoculation mycorhizienne comparativement au témoin et la meilleure amélioration a été obtenue avec l'inoculum M1.

\section{Evaluation de la biomasse aérienne, racinaire et totale}

Les résultats sur la biomasse aérienne, racinaire et totale sont présentés dans le
Tableau 3. Ces résultats sont variables en fonction des traitements. Les analyses statistiques montrent des différences significatives $(\mathrm{P}<0,05)$ entre les inoculés et le témoin pour tous les paramètres, mais il n'y a aucune différence significative entre les traitements inoculés. Les inoculations induisent généralement une amélioration de la biomasse comparée au témoin et tous les traitements inoculés ont des biomasses significativement supérieures à celle du traitement témoin. La meilleure amélioration est obtenue avec l'inoculum M1 pour la biomasse aérienne, racinaire et totale.

\section{Evaluation de la fréquence et de l'intensité de mycorhization}

Le Tableau 4 montre la fréquence et l'intensité de mycorhizien du sésame. La fréquence de mycorhization est assez élevée alors que l'intensité de mycorhization reste faible. Les analyses statistiques montrent des différences significatives $(\mathrm{P}<0,05)$ entre les différents traitements. Cependant, les racines du témoin ne sont pas mycorhizées.

Tableau 2 : Hauteur, taux de croissance relative en hauteur, diamètre au collet et taux de croissance relative du diamètre au collet du sésame inoculé avec deux souches de mycorhize indigène [complexe mycorhiziens (M1) et Glomus sp. (M2)].

\begin{tabular}{lllllll}
\hline Traitements & $\begin{array}{l}\text { Hauteur 1 } \\
(\mathbf{c m})\end{array}$ & $\begin{array}{l}\text { Hauteur 2 } \\
(\mathbf{c m})\end{array}$ & TCRh (\%) & $\begin{array}{l}\text { Diamètre 1 } \\
(\mathbf{m m})\end{array}$ & $\begin{array}{l}\text { Diamètre 2 } \\
(\mathbf{m m})\end{array}$ & TCRdc (\%) \\
\hline M1 & $17,4 \pm 0,94^{\mathrm{a}}$ & $63,75 \pm 2,47^{\mathrm{a}}$ & $2,68 \pm 0,1^{\mathrm{a}}$ & $3,24 \pm 0,19^{\mathrm{a}}$ & $5,12 \pm 0,94^{\mathrm{a}}$ & $0,57 \pm 0,23^{\mathrm{a}}$ \\
M2 & $17,17 \pm 1,3^{\mathrm{a}}$ & $60 \pm 2,89^{\mathrm{a}}$ & $2,51 \pm 0,1^{\mathrm{a}}$ & $3,12 \pm 0,29^{\mathrm{a}}$ & $4,95 \pm 0,83^{\mathrm{a}}$ & $0,57 \pm 0,15^{\mathrm{a}}$ \\
Témoin & $6,25 \pm 1,55^{\mathrm{b}}$ & $12 \pm 1,57^{\mathrm{b}}$ & $1,09 \pm 0,24^{\mathrm{b}}$ & $1,38 \pm 0,08^{\mathrm{b}}$ & $1,94 \pm 0,13^{\mathrm{b}}$ & $0,4 \pm 0,02^{\mathrm{a}}$ \\
$\begin{array}{l}\text { Niveau de } \\
\text { signification }\end{array}$ & $<0,001$ & $<0,001$ & $<0,001$ & $<0,001$ & 0,02 & $\mathrm{NS}$ \\
\hline
\end{tabular}

Témoin = non inoculé.

Hauteur 1 et 2 : hauteur mesurée respectivement à 30 et 60 jours après semis.

TCRh : taux de croissance relative en hauteur.

Diamètre 1 et 2 : diamètre mesuré respectivement à 30 et 60 jours après semis.

TCRdc : taux de croissance relative du diamètre au collet.

NS : non significatif.

Pour la même colonne (hauteur, taux de croissance relative en hauteur, diamètre au collet ou taux de croissance relative du diamètre au collet), les valeurs qui ont en commun une même lettre ne sont pas différentes significativement selon le test de Newman-Keuls au seuil de $5 \%$. 
Tableau 3 : Biomasse aérienne, racinaire et totale du sésame inoculé avec deux souches de mycorhize indigène [complexe mycorhiziens (M1) et Glomus sp. (M2)].

\begin{tabular}{lccc}
\hline Traitements & Biomasse aérienne (g) & Biomasse racinaire $(g)$ & Biomasse totale (g) \\
\hline M1 & $1,4 \pm 0,3^{\mathrm{a}}$ & $0,68 \pm 0,17^{\mathrm{a}}$ & $2,08 \pm 0,46^{\mathrm{a}}$ \\
M2 & $1,07 \pm 0,13^{\mathrm{a}}$ & $0,59 \pm 0,04^{\mathrm{a}}$ & $1,66 \pm 0,18^{\mathrm{a}}$ \\
Témoin & $0,69 \pm 0,06^{\mathrm{b}}$ & $0,3 \pm 0,03^{\mathrm{b}}$ & $0,99 \pm 0,07^{\mathrm{b}}$ \\
Niveau de signification & 0,006 & 0,008 & 0,006 \\
\hline
\end{tabular}

Témoin = non inoculé.

Pour la même colonne (biomasse aérienne, racinaire ou totale), les valeurs qui ont en commun une même lettre ne sont pas différentes significativement selon le test de Newman-Keuls au seuil de 5\%.

Tableau 4 : Fréquence et intensité de mycorhization du sésame inoculé avec deux souches de mycorhize indigène [complexe mycorhiziens (M1) et Glomus sp. (M2)].

\begin{tabular}{lcc}
\hline Traitements & Fréquence de mycorhization (\%) & Intensité de mycorhization $(\boldsymbol{\%})$ \\
\hline M1 & $95 \pm 5^{\mathrm{a}}$ & $28,78 \pm 3,11^{\mathrm{a}}$ \\
M2 & $73,33 \pm 3,33^{\mathrm{b}}$ & $15,77 \pm 1,97^{\mathrm{b}}$ \\
Témoin & $0^{\mathrm{c}}$ & $0^{\mathrm{c}}$ \\
Niveau de signification & $<0,0001$ & 0,003 \\
\hline
\end{tabular}

Témoin = non inoculé.

Pour la même colonne (fréquence et intensité de mycorhization), les valeurs qui ont en commun une même lettre ne sont pas différentes significativement selon le test de Newman-Keuls au seuil de $5 \%$.

\section{DISCUSSION}

Cette étude a été menée avec pour objectif d'évaluer la réponse du sésame à l'inoculation mycorhizienne avec des souches indigènes du Burkina Faso. Les résultats sur la mycorhization montrent que les racines du sésame sont fortement mycorhizées par les souches mycorhiziennes utilisées (Tableau 4). L'absence d'infection mycorhizienne sur les racines des témoins montre que les traitements sont exempts de toute contamination mycorhizienne. La stimulation de la croissance et de la biomasse entre les différents traitements et le témoin est attribuée à l'effet des champignons mycorhiziens arbusculaires inoculés.

Les résultats obtenus sur la croissance du sésame montrent que l'inoculation améliore la croissance de cette plante. Ainsi, l'inoculum M1 améliore la croissance en hauteur de $431,25 \%$, le taux de croissance relative en hauteur de $145,87 \%$, le diamètre au collet de $163,92 \%$, la biomasse aérienne de $102,9 \%$, la biomasse racinaire de $126,67 \%$ et la biomasse totale de $110,1 \%$ comparé au témoin non inoculé. Ces résultats pourraient s'expliquer par l'amélioration de la nutrition minérale du sésame par les souches symbiotiques inoculées. Cette amélioration par les champignons mycorhiziens arbusculaires (CMA) se manifeste d'abord par l'infection mycorhizienne puis par l'amélioration de la croissance et de la production de biomasse. Ces résultats corroborent ceux de Diouf et al. (2009) qui ont montré que l'inoculation mycorhizienne a permis d'augmenter la surface foliaire, le nombre de feuilles et la biomasse racinaire du sésame. Des résultats analogues ont été trouvés par Haro (2011) et Haro et al. (2012b) qui ont montré que l'inoculation mycorhizienne avec des souches indignes améliore mieux la croissance du niébé. De plus, les travaux de Haro et al. (2017) ont montré que l'inoculation avec les microorganismes indigènes améliore la production de biomasse aérienne et le rendement du niébé au même titre que la fertilisation avec les engrais chimiques (NPK), à la dose de $100 \mathrm{~kg} / \mathrm{ha}$. Ces 
résultats sont en concordance avec ceux de Ndoye et al. (2016) qui ont montré que l'inoculation avec les CMA G. verriculosum, $G$. manihotis et $R$. irregularis a amélioré significativement la biomasse des plants de fonio. Des résultats similaires ont également été trouvés par Ouahmane et al. (2007) qui ont montré les effets bénéfiques de la symbiose mycorhizienne sur la croissance de Cupressus atlantica. Aussi, les travaux de Ryan et Angus (2003) ont montré l'avantage des CMA pour l'augmentation de la productivité et l'approvisionnement en nutriments ( $\mathrm{P}$ et $\mathrm{Zn}$ ).

\section{Conclusion}

De ces résultats, il ressort de façon générale que l'inoculation mycorhizienne améliore la croissance et la production de biomasse du sésame. Cette étude a permis de montrer que les souches mycorhizienne natives du Burkina Faso améliorent aussi bien la production de biomasse que la croissance en hauteur. Ces souches natives ont montré des résultats prometteurs dans l'amélioration de la croissance du sésame. Cependant, il serait intéressant de tester la réponse à l'inoculation mycorhizienne de cette espèce avec les souches de la rhizosphère du sésame afin de sélectionner les CMA les plus efficaces. Aussi, il conviendrait de tester cette réponse à l'inoculation mycorhizienne en milieu réel et évaluer l'effet de ces inocula sur le rendement de cette espèce en vue de l'intégration de cette technique dans la culture du sésame pour l'amélioration de la productivité de celui-ci.

\section{CONFLIT D'INTERETS}

Les auteurs déclarent qu'ils n'ont aucun conflit d'intérêts.

\section{CONTRIBUTIONS DES AUTEURS}

$\mathrm{HH}$ a réalisé toutes les activités (conception, mise en place de l'essai, récolte et l'analyse des données et rédaction de ce manuscrit) de cette étude. SBK a été mise en sa mémoire pour lui rende hommage pour tout les sacrifices consentis pour notre encadrement de notre master et de notre thèse et jusqu'à son décès.

\section{REMERCIEMENTS}

Ce travail a bénéficié du soutien du Laboratoire Mixte International (LMI) « Intensification écologique des sols cultivés en Afrique de l'Ouest » (IESOL).

\section{REFERENCES}

Askari A, Ardakani MR, Paknejad F, Hosseini Y. 2019. Effects of mycorrhizal symbiosis and seed priming on yield and water use efficiency of sesame under drought stress condition. Scientia Horticulturae, 257: 108749. DOI: 10.1016/j.scienta.2019.108749

Boureima S. 2007. Influence de l'inoculation mycorhizienne arbusculaire sur la croissance et le developpement du Sesame (Sesamum indicum L.) au jeune âge. Diplôme d'Etudes Approfondies (DEA), Université Cheikh Anta Diop (UCAD). p. 57.

Diatta MB, Manzo OL, Diouf PRM, Diop T. 2013. Effets de l'inoculation mycorhizienne sur le sesame (Sesamum indicum L.) en conditions naturelles. Int. J. Biol. Chem. Sci., 7(5): 2050-2057. DOI: http://dx.doi.org/10.4314/ijbcs.v7i5.22

Diouf M, Bourema S, Diop TA. 2009. Reponses de deux varietes de Sesame a l'inoculation avec des champignons mychorhiziens arbusculaires candidats. Agronomie Africaine, 21(1): 37-47. DOI: http://dx.doi.org/10.4314/aga.v21i1.4620 0

FAO. 2019. Agricultural production, crop primary database. Food and Agricultural Organization of the United Nations, Rome.Agricultural production, crop primary database. Food and Agricultural Organization of the United Nations, Rome.

http://www.fao.org/faostat/fr/\#data/QC: Consulté le 10/2019.

Haougui A, Souniabe PS, Doumma A, Adam T. 2013. Evolution des populations des champignons endomycorhiziens sur les adventices de quatre sites maraîchers de la région de Maradi au Niger. Int. J. Biol. 
Chem. Sci., 7(2): 554-565. DOI: http://dx.doi.org/10.4314/ijbcs.v7i2.12

Haro H. 2011. Effet d'inoculums de champignons mycorhiziens arbusculaires sur la productivité du niébé Vigna unguiculata (L.) Walp. Diplôme d'Etudes Approfondies (DEA), Université de Ouagadougou. p. 94.

Haro H. 2016. Optimisation des symbioses rhizobienne et mycorhizienne pour améliorer la productivité du niébé [Vigna unguiculata (L.) Walp.] au Burkina Faso. Doctorat, Université Ouaga 1 Professeur Joseph Ki-Zerbo. p. 241.

Haro H, Sanon KB, Blagna F, Fofana B. 2016a. Effect of native arbuscular mycorrhiza fungi inocula on the growth of Cowpea [Vigna unguiculata (L.) Walp.] in three differents agro ecological zones in Burkina Faso. Journal of Applied Biosciences, 108: 10553-10560. DOI: http://dx.doi.org/10.4314/jab.v108i1.8

Haro H, Sanon KB, Diop I, Kane A, Dianda M, Houngnandan P, Neyra M, Traoré A. 2012a. Réponse à l'inoculation mycorhizienne de quatre variétés de niébé [Vigna unguiculata (L.) Walp.] cultivées au Burkina Faso et au Sénégal. Int. J. Biol. Chem. Sci., 6(5): 2097-2112. DOI: http://dx.doi.org/10.4314/ijbcs.v6i5.18

Haro H, Sanon KB, Krasova-Wade T, Kane A, N'Doye I, Traoré AS. 2015. Réponse à la double inoculation mycorhizienne et rhizobienne du niébé (variété, KVX3964-5-2D) cultivé au Burkina Faso. Int. J. Biol. Chem. Sci., 9(3): 1485-1493. DOI: http://dx.doi.org/10.4314/ijbcs.v9i3.31

Haro H, Sanon KB, Le Roux C, Duponnois R, Traoré AS. 2017. Improvement of cowpea productivity by rhizobial and mycorrhizal inoculation in Burkina Faso. Symbiosis: 1-14. DOI: 10.1007/s13199017-0478-3

Haro H, Sanon KB, Traoré AS 2012b. Réponse à l'inoculation mycorhizienne de deux variétés de niébé [Vigna unguiculata (L.) Walp.] cultivées au Burkina Faso. Les biotechnologies face aux défis du développement durable des états d'Afrique de l'ouest et du centre Ouagadougou (Burkina Faso).

Haro H, Sanon KB, Traoré AS. 2016b. Amélioration de la productivité du niébé par l'inoculation rhizobienne et mycorhizienne à Yakouta, Nord du Burkina Faso. Science et Technique, Sciences Naturelles et Agronomie, (2): 111-121.

Ndoye F, Diedhiou AG, Gueye M, Fall D, Barnaud A, Sy MO, Noba K, Diouf D, Kane A. 2016. Réponse du fonio blanc (Digitaria exilis Stapf) à l'inoculation avec des champignons mycorhiziens à arbuscules en conditions semi-contrôlées. Journal of Applied Biosciences, 103(1): 9784-9799.

DOI: http://dx.doi.org/10.4314/jab.v103i1.1

Ouahmane L, Thioulouse J, Hafidi M, Prin Y, Ducousso M, Galiana A, Plenchette C, Kisa M, Duponnois R. 2007. Soil functional diversity and $\mathrm{P}$ solubilization from rock phosphate after inoculation with native or allochtonous arbuscular mycorrhizal fungi. Forest Ecology and Management, 241(1-3): 200-208. DOI: 10.1016/j.foreco.2007.01.015

Phillips JM, Hayman DS. 1970. Improved procedures for clearing roots and staining parasitic and vesicular-arbuscular mycorrhizal fungi for rapid assessment of infection. Transactions of the British Mycological Society, 55(1): 158-161.

Ryan MH, Angus JF. 2003. Arbuscular mycorrhizae in wheat and field pea crops on a low P soil: increased $\mathrm{Zn}$-uptake but no increase in P-uptake or yield. Plant and Soil, 250(2): 225-239. DOI: 10.1023/a:1022839930134

Trouvelot A, Kough JL, Gianinazzi-Pearson V, Gianinazzi S. 1986. Mesure du taux de mycorhization VA d'un système radiculaire. Recherche de méthodes d'estimation ayant une signification fonctionnelle. Mycorrhizae : Physiology and Genetics: 217-221. 